ISSN: 0212-0267

DOI: http://dx.doi.org/Io.I420I/hedu20I534263286

\title{
LA CANCIÓN ESCOLAR EN ESPAÑA ENTRE I900 Y 1936. CONTRIBUCIÓN AL ESTUDIO HISTÓRICO DE LA EDUCACIÓN MUSICAL
}

\section{Classroom songs in Spain between 1900 and 1936. A contribution to the bistorical study of musical education}

Consuelo de la Vega Sestelo

Universidad de Salamanca. Dpto. de Educación Musical, Plástica y Corporal

Correo-e: vegasestelo@usal.es

Recepción: ro de julio de 20. Envío a informantes: 20 de septiembre de 2014 .

Fecha de aceptación definitiva: 16 de abril de 2015

Resumen: Este trabajo se centra en el estudio y análisis de canciones infantiles y escolares destinadas a la enseñanza primaria, en España, entre 1900 y 1936, y la formación recibida por los maestros a través de los métodos empleados en la enseñanza de la música en las Escuelas Normales. De forma analítica y comparativa se determinan las características de las composiciones, desde el punto de vista musical y didáctico, y la formación de los maestros para utilizarlas en la escuela. Se obtienen conclusiones que nos acercan al conocimiento detallado de una canción escolar, de sus características, de sus dificultades de aprendizaje, poniéndonos de manifiesto la importancia de estas pequeñas obras en la enseñanza primaria, así como la escasa preparación musical de los maestros para poder utilizarlas.

PALABRAs Clave: canción infantil; canción escolar; educación musical; enseñanza primaria; formación de maestros; manual.

AвstRAct: This work is focused on the study and analysis of nursery and primary school songs in Spain in the period from 1900 to 1936 and the education in music received by teachers through the music teaching methods at the 'Normal Schools', as teachers' colleges were called. The musical and educational characteristics of these compositions, and how teachers were taught to use them in the 
classroom, are determined through methods of analysis and comparison. The conclusions reached provide a detailed understanding of classroom songs, their features and their learning difficulties, highlighting the importance of these minor works in primary education, as well as teachers' poor musical knowledge regarding how to use them.

KEY WORDs: nursery song; classroom song; musical education; primary education; teacher training; handbook.

\section{Introducción}

$\tau$

A Música COMO MATERIA FACTIBLE DE SER ENSEÑADA, como herramienta que puede ser utilizada en la educación en general y en particular de los más pequeños, como área de conocimiento que desarrolla su propio avance intelectual y científico, como lenguaje artístico que puede acompañar a las personas a lo largo de su vida, como desarrollo del sentimiento estético merece un estudio específico, una investigación detallada, estructurada y rigurosa de las múltiples facetas que la componen.

Siendo conscientes del amplio campo de trabajo que ofrece el mundo de la música, y lo poco abordado que se encuentra, sobre todo en su enseñanza y su historia, limitamos estas páginas al trabajo realizado sobre la canción infantil utilizada en la enseñanza dirigida a la infancia como canción escolar, a través de su historia en la educación musical y educación integral de los más pequeños; y al estudio de la formación recibida por los maestros en esta área de conocimiento entre 1900 y 1936 en España. Este estudio se centra en el análisis realizado a 782 canciones infantiles escritas o armonizadas para ser utilizadas como canciones escolares y en 36 métodos de música destinados a Escuelas Normales.

El motivo intelectual y científico de la elección de este periodo hace referencia a que, durante este tiempo, en la primera enseñanza, permanece en vigor el plan de estudios de $190 \mathrm{I}^{\mathrm{I}}$ donde, en sus tres grados: de párvulos, elemental y superior, se incluye entre sus materias la de Canto. Conocer el material o canciones que se pudieron utilizar en esta enseñanza, conocer los motivos que llevaron a que su composición tuviese unas determinadas características nos ofrece importante y abundante información sobre músicos que participaron en este empeño, enseñantes y profesores preocupados por los maestros que llevarían a cabo la labor de enseñar estas canciones y sobre el pensamiento educativo de los profesionales de la educación musical, no siempre valorados o tenidos en cuenta en el desarrollo de los sistemas educativos nacionales. Este terreno de estudio ha sido escasamente abordado desde el punto de vista histórico, musical y didáctico. Ahondar en parte de esta historia nos acerca

Ministerio de Instrucción Pública y Bellas Artes: «Real Decreto de 26 de octubre de I90I, autorizando al Ministro de Instrucción Pública y Bellas Artes para que en los presupuestos generales de su departamento, a partir del que se forme para 1902, incluya las partidas necesarias, conforme a las disposiciones de este decreto, para el pago de las atenciones de personal y material de las Escuelas públicas de primera enseñanza», Gaceta de Madrid de 30 de octubre de 1901, n. ${ }^{\circ} 303$, pp. 497-499. 
LA CANCIÓN ESCOLAR EN ESPAÑA ENTRE I9OO Y 1936. CONTRIBUCIÓN AL ESTUDIO HISTÓRICO DE LA EDUCACIÓN MUSICAL CONSUELO DE LA VEGA SESTELO

a la construcción de la historia de la educación musical en España y de lo que ello significa para conocer el porqué de la educación musical actual.

\section{La canción infantil como documento histórico educativo. La canción escolar}

Reflexionar sobre un documento educativo, su historia, sus características, su situación en la sociedad, sus objetivos, sus circunstancias, sus receptores, sus creadores, las aportaciones recibidas en su utilización, sus transmisores, etc., es tarea siempre difícil en cualquier caso, pero especialmente complicada si nos referimos a la canción infantil; documento donde intervienen aspectos compositivos, constructivos, didácticos, sonoros, temporales, culturales, estéticos y sociales adentrándonos en el estudio de su función y contribución al desarrollo de un grupo humano, una nación, una sociedad, una ideología o una transmisión de valores ciudadanos.

¿Qué es una canción escolar? Llegar a elaborar una definición es tener en cuenta aspectos sociales que han llevado a su creación, aspectos y características de los destinatarios, intenciones y objetivos educativos con su utilización, aspectos compositivos musicales puestos al servicio de este tipo de obras y sobre todo ¿las acepta la propia infancia?, son ellos los que a través del tiempo eligen, sin entrar en detalles del porqué de esta elección, el tipo de canciones y música que les gusta, les reconforta, les hace tomar conciencia de grupo, y que recogen, como fuente para ser utilizada en la educación, los distintos profesionales en cada época de la historia.

Definimos la canción escolar como una obra musical de pequeño formato destinada a la infancia con un fin educativo y didáctico.

A través del estudio realizado en la época señalada, se pretende dejar constancia de que las canciones infantiles poseen unas características determinadas para poder ser consideradas escolares, que fueron utilizadas con fines educativos y fueron fruto de aplicaciones didácticas concretas que avalan el porqué de su uso actual en la enseñanza primaria y en la formación de los maestros. Con las conclusiones obtenidas en esta época se quiere iniciar un estudio histórico y evolutivo de la canción escolar y de las características que perduran o varían a través del tiempo.

¿Por qué es importante la canción desde el punto de vista escolar? ¿Por qué resulta imprescindible su estudio y conocimiento desde cualquier punto de vista incluido el histórico o el musical? Para poder contestar a estas preguntas vamos a reflejar lo que Leticia Sánchez especifica sobre el canto coral en la Institución Libre de Enseñanza ${ }^{2}$ que utilizamos como resumen de las opiniones pedagógicas más importantes del primer tercio del siglo xx en España. La aplicación de muchos de los ideales pedagógicos institucionistas y krausistas lo permitiría el empleo del canto coral, como son:

I. Logro de la práctica activa de la música, educando el sentimiento estético que permitía incidir sobre el gusto artístico natural del niño.

Cfr. SÁnchez de Andrés, Leticia: Música para un ideal. Pensamiento y actividad musical del Krausismo e Institucionismo españoles (I854-I936), Madrid, SEM, 2009, pp. 287-289. 
2. Herramienta útil para mediatizar otros aprendizajes.

3. Propiciar el aprendizaje intuitivo.

4. Estimular la sociabilidad del individuo y su capacidad creativa.

5. Permitir la educación armónica e integral de cuerpo y espíritu (razón y sentimiento) a través de la práctica de la música vocal.

6. Favorecer el desarrollo europeísta y moderno.

7. Considerar el canto coral como una actividad física y formativa de la razón y del sentimiento.

8. Ayudar al equilibrio entre la salud del cuerpo y del espíritu.

9. Permitir el trabajo sobre la higiene del aparato fonador y respiratorio.

Io. Educar los sentidos y transformar el cuerpo humano, en su sentido más íntimo, en un instrumento artístico.

II. Cultivar la voluntad a través del trabajo en comunidad.

I2. El canto estimula la sociabilidad y la unión entre los alumnos de la Institución.

La canción escolar es, por sí misma, un objeto de estudio específico. Importante por lo que significa para la educación integral del niño; importancia que se sigue considerando actualmente en la enseñanza primaria y como objetivo en la preparación de los maestros.

\section{Reseña histórica sobre el canto y la canción en la educación musical de la infancia}

Desde los tiempos más remotos de la existencia del hombre encontramos un paralelismo entre el lenguaje hablado y cantado como dos manifestaciones de comunicación con objetivos y efectos claramente definidos. Todo pueblo o cultura ha poseído y posee cantos apropiados a su naturaleza, a su instinto y a su cultura.

En la Antigüedad, Platón como uno de los filósofos más representativos del pueblo griego, defendía la utilización de la música antes que cualquier otra arte para la formación de los hombres, formando antes el alma que el cuerpo 3 :

- Ahora bien, Glaucón, la educación musical es de suma importancia a causa de que el ritmo y la armonía son lo que más penetra en el interior del alma y la afecta más vigorosamente, trayendo consigo la gracia, y crea gracia si la persona está debidamente educada, no si no lo está. Además, aquel que ha sido educado musicalmente como se debe es el que percibirá más agudamente las deficiencias y la falta de belleza, tanto en las obras de arte como en las naturales, ante las que su repugnancia estará justificada.

En la Península Ibérica los pueblos foráneos como fenicios, hebreos, griegos y cartagineses se fueron asentando a partir del siglo viri a. de C. Aportaron su

Platón: Diálogos. IV La República. Introducción, traducción y notas de Conrado Eggers Lan, Madrid, Editorial Gredos, 1998, Libro III 398c a 403c (fragmento). 
LA CANCIÓN ESCOLAR EN ESPAÑA ENTRE I9OO Y I936. CONTRIBUCIÓN AL ESTUDIO HISTÓRICO DE LA EDUCACIÓN MUSICAL CONSUELO DE LA VEGA SESTELO

cultura, entre ella la musical, que podemos suponer acompañada de una forma de enseñanza y de aprendizaje ${ }^{4}$. Esto mismo ocurriría con la romanización de la Península Ibérica. La entrada del cristianismo en la Península, con su origen en las primeras comunidades judías, aporta prácticas y rituales donde interviene la música, fusionando aspectos romanos e indígenas. En estos primeros siglos existieron niños cantores asociados a los cultos religiosos, por tanto, podríamos hablar de un nivel de educación musical de ellos.

En el aprendizaje musical de la infancia, en estos siglos, debemos tener en cuenta dos vertientes: en primer lugar, existe un desarrollo educativo relacionado con el culto religioso; y, en segundo lugar, se manifiesta un aprendizaje popular de transmisión oral donde los niños participarían de esta actividad con sus cantos y juegos.

En el siglo viri llegan los musulmanes a la península. En esta ocasión el arte musical era practicado por mujeres y niñas, al contrario que en el cristianismo que solo lo era por hombres o niños.

La educación musical en la Iglesia adquiere cada vez mayor importancia; así, entre los siglos XI y XV, aparecen en España prestigiosos centros monásticos y catedralicios donde se imparte una esmerada enseñanza musical. Se educaba infantes formando grupos de niños de coro. Estas prácticas musicales y religiosas se extienden por todas las iglesias con la llegada de la polifonía desde el siglo XVI al XVIII y principios del XIX.

De forma paralela, la población en general, sobre todo aquella sin formación, participa y es agente que interviene y recibe los cantos, juegos y manifestaciones culturales por medio de la transmisión oral. Estructuras y secuencias que pasan de padres a hijos, siendo los niños receptores y transmisores en la edad adulta de estas canciones.

Las nuevas ideas de la Ilustración referidas a la enseñanza se ven reflejadas en España en la reforma educativa del conde de Aranda, que pronto fundó escuelas en muchas zonas rurales. En estas escuelas la música se utilizaba de manera accesoria, como canciones populares con las que los alumnos aprendían las tablas de multiplicar, los nombres de los ríos, etc. No era una formación musical como tal sino que se utilizaba la música como una herramienta metodológica que a la vez servía de divertimento y de forma más entretenida de aprendizaje.

La Europa de finales del siglo XIX y principios del xx se caracteriza por la diversidad de las clases sociales. La música se cultiva, por una parte de forma erudita, con mayor complejidad de comunicación; y, en otras, en la población más ignorante, por medio de canciones populares que se transmiten de unos a otros. En España, en el siglo xix se produce la decadencia de la enseñanza musical en los centros eclesiásticos.

Establecer la importancia y trascendencia de la música en las escuelas en la época tratada, lo que se consideraba como Pedagogía Moderna es algo que podemos

4 Cfr. SÁNChez López, Gustavo: «Apuntes históricos sobre la enseñanza musical en España», en Ministerio de Educación, Política Social y Deporte: La música como medio de integración y trabajo solidario, Madrid, Secretaría de Estado de Educación y Formación, 2008, pp. 25-50. 
LA CANCIÓN ESCOLAR EN ESPAÑA ENTRE I9OO Y I936. CONTRIBUCIÓN AL ESTUDIO HISTÓRICO DE LA EDUCACIÓN MUSICAL CONSUELO DE LA VEGA SESTELO

constatar como un ideal perseguido por pedagogos como Pestalozzi, Fröebel, el P. Girard, Bassedow o Compayré; por sociólogos como Bain y Spencer; por compositores como Saint-Saëns o grandes profesores de música como Gavín, Chevé y Danhausse. Autores como Orff, Dalcroze, Kodaly o Willems desarrollan su actividad pedagógica y musical en la primera mitad del siglo xx y años posteriores. Sus teorías se centran en la canción infantil como eje conductor de las actividades musicales con los niños. Estas corrientes llegan a España, aunque el abandono de esta enseñanza en las escuelas y la inferioridad respecto al resto de los países europeos es evidente. En I902, Vancells comenta:

Es indudable que los niños son antes seres sensibles que inteligentes, y que a la sensibilidad hay que dirigirse para despertar su inteligencia [...]. Bien lo han entendido así los alemanes, que desde el siglo pasado consideran obligatoria la enseñanza de la música en las escuelas, París desde principios de siglo [siglo XIX], toda Francia desde el año $27,[\ldots]$. Sólo nosotros nos empeñamos en no ver el valor educativo en general de la Música y el Canto; [...].

En España no se da la trascendencia que la música tiene en la educación de la infancia, pero sí existían voces en defensa del desarrollo del canto en las escuelas y la necesidad de una preparación adecuada de los maestros. Músicos como Martínez Torner, Rafael Benedito, Manuel Borguñó, Rogelio Villar, Juan Llongueras, J. Salvador Martí, etc., así lo hacen constatar en numerosos escritos. Rogelio Villar $^{6}$ en I9I2 $^{7}$ manifiesta:

Pero más pesadumbre sentíamos cuando, al ver lo poco que aquí se hace en estas materias [música, canto], la ignorancia que reina sobre ellas y el poco interés que se toman los Gobiernos por los problemas de la educación [...].

La España educada, avanzada, la que recogía los nuevos planteamientos pedagógicos es consciente de estas nuevas ideas, de lo que debe ser la educación de un niño. José Salvador ${ }^{8}$ dice en su libro La nueva enseñanza de la música9

Yo busco una escuela nueva, original, con personalidad propia, de acuerdo con nuestro temperamento meridional, vivo, creador, artista. Una enseñanza que no sea diluida y fatigante, atrofiadora o destructora de nuestra personalidad, sino al contrario, que la desenvuelva ampliamente, educando física, intelectual y estéticamente

Juan Vancell y Roca fue profesor de Música de la Escuela Normal de Maestros de Barcelona, y que tengamos constancia, al menos, entre 1902 y 1920. VANCELl y Roca, Juan: El libro de Música y Canto, Barcelona, Fidel Giró, 1902, prólogo, p. I.

6 Rogelio del Villar González (1875-1937). Compositor, crítico, folklorista y publicista docente. Conocido crítico y musicógrafo, fue catedrático de Música de Cámara del Real Conservatorio de Música de Madrid. Fundador en 1928 de la revista Ritmo.

VIllar, Rogelio: La música en las escuelas, Madrid, El Magisterio español, 1912, pp. 5-6.

8 José Salvador Martí (I874-1947). Pianista valenciano, compositor, pedagogo, escritor y pensador musical. Sus planteamientos pedagógicos giraban en torno a la idea de dividir la enseñanza musical en dos vertientes, coexistiendo ambas: una específica impartida en conservatorios y otra insertada en el plan general de estudios español.

9 Salvador Martí, José: La nueva enseñanza de la música, Valencia, Mundial Música, 1932, Pp. $2 \mathrm{I}-22$. 
LA CANCIÓN ESCOLAR EN ESPAÑA ENTRE I9OO Y I936. CONTRIBUCIÓN AL ESTUDIO HISTÓRICO DE LA EDUCACIÓN MUSICAL CONSUELO DE LA VEGA SESTELO

al alumnado según sus condiciones particulares y nativas; que el estudio resulte, además, de consciente, fácilmente comprensible, ameno, interesante, animado, lleno de vida, y permita colocar al discípulo en todos los casos en condiciones de pensar, juzgar y expresar de por sí. [...].

La situación de la educación musical en la enseñanza primaria y Escuelas Normales en España a finales del siglo xix y principios del xx es muy deficiente. Considerada la música como una de las artes más populares, es la más cenicienta de todas cuando se trata de desarrollar su labor pedagógica. A pesar de esta situación, es verdad que se consideraba necesario llevar la música a la escuela a través del aprendizaje de los cantos escolares. Fruto de esto son las publicaciones que nos encontramos con canciones infantiles y escolares, desarrollo de un material pedagógico al alcance de los maestros o creado para ellos. No podemos constatar la totalidad de la utilización del material analizado, pero sí podemos dejar constancia de la existencia de esos materiales, de sus características, de las voces que afirman la necesidad de la educación musical dentro de la educación integral del niño y de la formación de los maestros para utilizarlas.

\section{La educación musical en la Enseñanza Primaria en España entre 1900 y 1936}

Revisando la normativa referida a la educación musical y cantos escolares en la Enseñanza Primaria en España entre 1900 y 1936 nos daremos cuenta de la importancia, o no, que los poderes legislativos otorgaban a este aspecto de la educación.

\section{I. La situación de la materia de música anterior a Igoo}

Anteriormente a I900, todo está referido al canto escolar como una actividad educadora, buena para la infancia, para su desarrollo personal, su equilibrio mental y físico y como aportación a una enseñanza estética de lo bello y del buen gusto, y no, como una materia o asignatura del plan de estudios.

La Ley de Instrucción primaria de $1868^{10}$, ley con corta vida, derogada pocos meses después de su aprobación ${ }^{\text {II }}$, hace constar que los niños y niñas ejerciten el canto en todas aquellas escuelas en que hubiese medios para ello. El Reglamento ${ }^{12}$ que desarrolla la Ley divide todas las materias en tres grados y especifica que el canto, al igual que las demás, se limitará a lo más esencial. En el caso de párvulos

1o Ministerio De Fomento: «Ley de Instrucción primaria de 2 de junio de i868», Gaceta de Madrid de 4 de junio de I868, n. ${ }^{\circ}$ 156, pp. I-4, art. I4.

i MinisTERIO DE FOMENTO: «Decreto de I4 de octubre de I868, derogando la Ley de Instrucción primaria y el Reglamento para ejecutarla, declarando libre la enseñanza primaria, restableciendo las Escuelas Normales y más que expresa», Gaceta de Madrid de Is de octubre de i868, n. ${ }^{\circ}$ 289, pp. 4-5.

${ }_{12}$ Ministerio de Fomento: «Real Decreto de io de junio de i868, aprobando el Reglamento de Instrucción primaria», Gacetas de Madrid de 17 de junio de I868, n. ${ }^{\circ}$ I69, pp. 2-6; de I8 de junio de I868, n. ${ }^{\circ}$ I70, pp. 3-IO; de I9 de junio de I868, n. ${ }^{\circ}$ I7I, pp. 2-5; y de 20 de junio de I868, n. ${ }^{\circ}$ I72, pp. 7-9, arts. 300 y 305 . 
LA CANCIÓN ESCOLAR EN ESPAÑA ENTRE I9O0 Y 1936. CONTRIBUCIÓN AL ESTUDIO HISTÓRICO DE LA EDUCACIÓN MUSICAL CONSUELO DE LA VEGA SESTELO

enumera una serie de ejercicios: marchas, evoluciones y movimientos ejecutados a compás por los discípulos, en común, cantando o en silencio; cantos religiosos y morales de corta extensión.

Nos trasladamos al año i898, con la derrota sufrida por España en Cuba. El país siente la necesidad de la regeneración, que se asocia, entre otras cosas, a la implantación de un nuevo modelo de organización escolar: la escuela graduada. Esta forma de estructurar la enseñanza ya era habitual en otros países y, según sus seguidores, respondía a las exigencias de la Pedagogía Moderna convirtiéndose así en un signo de europeización y paradigma de la nueva escuela nacionalin. En las escuelas graduadas anejas a las Escuelas Normales se determina por medio de un Reglamento de $1899^{14}$ la obligatoriedad de la enseñanza de cantos sencillos en todas las secciones.

Es importante la introducción de este tipo de escuelas en España. Antonio Viñao $^{\text {Is }}$ especifica que no hablamos de graduación escolar, sino de la escuela graduada como forma organizativa que va más allá de los métodos; como comenta Cossío $^{16}$ se trata de agrupar a los niños por edades y grados de cultura, formando verdaderas clases homogéneas, encomendando cada una de ellas a un maestro. Concebidas las escuelas graduadas anejas como escuelas modelo, servirían para llevar a la práctica de forma experimental las nuevas ideas pedagógicas, entre ellas, la obligatoriedad de los cantos en la enseñanza.

A pesar de lo anterior, en 1900 se aprueba un nuevo Reglamento orgánico ${ }^{17}$ de primera enseñanza, donde no se cambia, ni siquiera se trata, en su fundamentación, la posibilidad de modificar el plan de estudios de la enseñanza primaria.

Como resumen de la situación dejamos constancia con las palabras que en I899 expresa Francisco J. Cobos, director de la Escuela Normal de Granada ${ }^{18}$ :

[...] en casi todas las naciones ilustradas la Música forma parte del programa de primera enseñanza, y los niños se acostumbran á leer en el pentagrama [...]. Aún no hemos adelantado tanto nosotros en el camino de la cultura general; pues aparte de las escuelas Normales centrales, de las de sordo-mudos y ciegos de Madrid y Barcelona, y de algunas otras muy contadas, la enseñanza de la Música es todavía una esperanza, no fácilmente realizable, en la instrucción primaria.

${ }^{13}$ Cfr.: ViÑaO Frago, Antonio: «La escuela graduada: una nueva organización escolar y pedagógica», en Álvarez Lázaro (dir.): Cien años de Educación en España, Madrid, Ministerio de Educación, Cultura y Deportes, Fundación BBVA, 200I, pp. 363-367.

${ }_{14}$ Ministerio de Fomento: «Real Decreto de 29 de agosto de 1899 , fijando el reglamento por que han de regirse las Escuelas Graduadas á que se refieren los artículos $2 .^{\circ}, 3 .^{\circ}, 4 .^{\circ}$ y $5 .^{\circ}$ del Real Decreto de 23 de septiembre de I898», Gaceta de Madrid de 5 de septiembre de 1899 , n. ${ }^{\circ}$ 248, pp. 863-865.

is VIÑNAO FRAGO, Antonio: Innovación pedagógica y racionalidad científica. La escuela graduada pública en España (I898-1936), Madrid, Ediciones Akal, I990, p. 8.

16 Citado en: Viñao Frago, Antonio: 200I, op. cit., p. 363.

${ }_{17}$ Ministerio de Instrucción Pública y Bellas Artes: «Real Decreto de 6 de julio de i9oo, aprobatorio del reglamento orgánico de primera enseñanza», Gaceta de Madrid 8 de julio de i90o, n. ${ }^{\circ}$ I89, pp. II6-II8.

${ }_{18}$ En la Carta-Prólogo de: Fuentes Aguilera, Sebastián: La música para los párvulos, Granada, Imprenta de la Viuda e hijos de P. V. Sabadell, I899. 
LA CANCIÓN ESCOLAR EN ESPAÑA ENTRE I9OO Y I936.

CONTRIBUCIÓN AL ESTUDIO HISTÓRICO DE LA EDUCACIÓN MUSICAL

\subsection{El plan de estudios de la primera enseñanza de IgoI}

Es la situación precaria de la primera enseñanza y de los maestros hasta este momento lo que hace que el ministro de Instrucción Pública, Álvaro Figueroa, conde de Romanones, aborde la búsqueda de soluciones en un Real Decreto de I9OI $^{19}$, regulando, a la vez, un nuevo plan de estudios de la enseñanza primaria. Recoge el testigo de la enseñanza de cantos sencillos ya implantados de forma experimental en las escuelas graduadas anejas. La primera enseñanza pública establecida en tres grados: párvulos, elemental y superior, incluye entre sus materias la de Canto. Al igual que el resto de las asignaturas se impartirá en todos los cursos, distinguiéndose únicamente por la amplitud del programa y por el carácter pedagógico y duración de sus ejercicios.

\subsection{La legislación en los años posteriores a IgoI}

Después del plan de estudios de I90I, que duraría muchos años, se producen intentos de redactar nuevas leyes de educación que sustituyan a la vigente de i857. En lo que se refiere a la enseñanza primaria, el Proyecto de ley de $1902^{20}$, refleja la necesidad de simplificar las múltiples disposiciones, resolviendo así las incertidumbres que esta situación ha provocado; intentar unificar los criterios legislativos que ayudasen a unificar a su vez los criterios pedagógicos; incluye el Canto entre las materias de la primera enseñanza. Un segundo Proyecto de ley de bases se produce en $1903^{21}$, en este caso no se incluye ni la Música ni el Canto entre las materias obligatorias del plan de estudios. El siguiente proyecto de $1905^{22}$ vuelve a incluir el Canto entre las materias de estudio.

La asignatura de Canto no era considerada como una materia fundamental dentro del currículum de la primera enseñanza, tal como se puede deducir de una Instrucción de $1908^{23}$, de la Junta Central de primera enseñanza, donde se estructura la forma de los exámenes que han de celebrarse en las Escuelas públicas. En estos exámenes no se incluyen contenidos correspondientes a la enseñanza del

19 Ministerio de Instrucción Pública y Bellas Artes: «Real Decreto de 26 de octubre de I90I», op. cit.

${ }_{20}$ Ministerio de Instrucción Pública y Bellas Artes: «Real Decreto de 17 de octubre de 1902, autorizando al Ministro de Instrucción Pública para presentar a las Cortes el proyecto de ley de bases de reorganización de la enseñanza», Gaceta de Madrid de 5 de noviembre de 1902, n. ${ }^{\circ} 309$, pp. $419-42 \mathrm{I}$.

${ }_{21}$ Ministerio de Instrucción Pública y Bellas Artes: «Real Decreto de 29 de mayo de I903, autorizando al Ministro de Instrucción Pública para someter a la deliberación de las Cortes un proyecto de ley de Bases de la enseñanza en general y de reorganización de la primaria», Gaceta de Madrid de i de junio de 1903 , n. ${ }^{\circ}$ I52, pp. 815-818.

22 Ministerio de Instrucción Pública y Bellas Artes: «Real Decreto de i4 de junio de I905, autorizando al Ministro de Instrucción Pública para someter á deliberación de las Cortes un proyecto de ley orgánica de instrucción primaria», Gaceta de Madrid de I6 de junio de 1905, n. ${ }^{\circ}$ I67, pp. 1089-1092.

${ }_{23}$ Junta Central de PRIMERA enseñanza: «Instrucción de 25 de mayo de 1908, relativa al programa para los exámenes que han de celebrarse en las Escuelas públicas», Gaceta de Madrid de 3I de mayo de I908, n. ${ }^{\circ}$ I52, pp. IO30-IO3I. 
LA CANCIÓN ESCOLAR EN ESPAÑA ENTRE I9OO Y 1936. CONTRIBUCIÓN AL ESTUDIO HISTÓRICO DE LA EDUCACIÓN MUSICAL CONSUELO DE LA VEGA SESTELO

Canto. Sí se establece que al terminar el acto académico se interpretarán cantos escolares, principalmente religiosos y patrióticos, constatando así su consideración como un complemento en la educación.

El plan de estudios de I90 tiene una larga duración, no cambiando en el periodo que nos ocupa de 1900 a 1936 . La preocupación por mejorar la enseñanza popular fue siempre una constante en los sucesivos y «masivos» cambios de ministros de Instrucción Pública, aunque centrados más en la reforma del Magisterio como solución a los males que padecía la enseñanza.

Iniciada la II República en 1931, se crea, dependiente del Ministerio de Instrucción Pública, un Patronato de Misiones Pedagógicas. Se intenta poner en marcha las nuevas ideas de la República que consideran a la educación como un motor de cambio social. Se quiere llegar sobre todo a las zonas rurales. Entre los trabajos encomendados está el de establecer sesiones musicales de coros y pequeñas orquestas cuando sea posible, y en todo caso de audiciones por radiotelefonía y discos cuidadosamente seleccionados.

\section{La formación musical de los maestros en España entre 1900 y 1936}

\section{I. La situación anterior a I898. El último tercio del siglo XIX}

Es el siglo xviII, el siglo del pensamiento ilustrado en Europa, y con él la aparición de la necesidad de conseguir la universalización de la enseñanza. Pero, en España, este espíritu de la Ilustración, en la educación, no lo encontramos reflejado en auténticas disposiciones hasta comienzos del siguiente siglo.

La Constitución de $1812^{24}$ establece que la enseñanza debe ser uniforme para todos los ciudadanos, universal y gratuita. En i813 se realiza un proyecto de normativa general, el Informe Quintana. Pese a no ser discutido en Cortes, ni aprobado, fue decisivo en años posteriores.

En el periodo de la monarquía absolutista la educación experimenta un retroceso. A la muerte del monarca Fernando VII, la preocupación era la de encontrar una estabilidad política. Ante esta situación se intenta retomar el tema de la educación con un proyecto en I838 atribuido a Montesino, que tampoco fue aprobado, y con el Plan Pidal, esta vez aprobado con rango de Decreto en $1845^{25}$.

El primer texto educativo legal que recoge los planteamientos de la Constitución de I8ı2 y que venía a poner estabilidad en un sistema de enseñanza es la Ley Moyano de $1857^{26}$. Regula los distintos niveles de enseñanza, tipos de escuelas, su creación, las titulaciones oficiales, saberes elementales y específicos, estructuración

24 Cortes Generales de España: Constitución Política de la Monarquía Española, promulgada el ı9 de marzo de i8ı2, Cádiz, ı8ı2.

25 Ministerio de la Gobernación: «Real Decreto de 17 de septiembre de i845, aprobando el plan general de estudios para la instrucción pública del reino en la parte relativa á las enseñanzas secundaria y superior», Gaceta de Madrid de 25 de septiembre de 1845, n. $^{\circ} 4029$, pp. I-5.

26 Ministerio De Fomento: «Ley de 9 de septiembre de i857, de Instrucción pública autorizada por el Gobierno para que rija desde su publicación en las Península é Islas adyacentes, lo que se cita», Gaceta de Madrid de Io de septiembre de I857, n. ${ }^{\circ}$ I710, pp. I-3. 
LA CANCIÓN ESCOLAR EN ESPAÑA ENTRE I9OO Y I936. CONTRIBUCIÓN AL ESTUDIO HISTÓRICO DE LA EDUCACIÓN MUSICAL CONSUELO DE LA VEGA SESTELO

administrativa de la enseñanza, etc. ${ }^{27}$. De una u otra forma esta ley ha estado siempre de base en todos los movimientos de organización educativa del Estado hasta bien entrada la segunda mitad del siglo xx. Dentro de este marco, en el año I870, se presenta a las Cortes una proposición de Ley sobre primera instrucción para España e islas adyacentes. En ella ya se contempla la necesidad de que en las Escuelas Normales exista un propietario Maestro de Música y Canto y una propietaria Maestra de Música y Canto ${ }^{28}$.

A pesar de los intentos por renovar la enseñanza, incorporando las nuevas ideas de la filosofía krausista, este final de siglo iba a ser un periodo de cierto abandono de las Escuelas Normales. Nos comentan Ávila y Holgado ${ }^{29}$ :

Las Escuelas Normales sufren también este signo de los tiempos, la despreocupación, donde la falta de entusiasmo a la par que una legislación nada sustancial van a provocar el lamentable nivel de estos centros de formación del magisterio tanto masculino como femenino.

Es en I878 cuando una primera disposición en España por medio de una Real Orden ${ }^{30}$ establece, por vía de ensayo, en las Escuelas Normales Centrales de Madrid, la implantación de la asignatura de Música y Canto en los planes de estudio, para más tarde poder hacerlo extensivo al resto de las Normales de las provincias. Las lecciones serían diarias, con una duración de una hora, por lo menos. El primer curso consistiría en ejercicios de canto a oído y elementos del solfeo.

Son muchas las voces que consideran la necesidad de impartir la asignatura de Música y Canto a los futuros maestros. Como ejemplo en el libro de Agustín M. Lerate $^{3 \mathrm{I}}$ (I882) Abecedario musical ó Cartilla de Música y Canto: escrita expresamente para las Escuelas de Primera enseñanza $a^{32}$, la siguiente dedicatoria:

Á la Ecxma. Diputación Provincial de Cádiz. [...] Á mayor abundamiento, V. E. costea de su presupuesto la Escuela Normal de Cádiz, primera de España que estableció clase de música para sus alumnos, [...] fué [sic] también la primera en presentir los propósitos del Gobierno, claramente manifestados más tarde al establecer la asignatura de la música y canto en las Escuelas Normales de Madrid, como preparación para hacerla luego estensiva [sic] á todas las escuelas de primera enseñanza de la nación [...].

${ }_{27}$ Cfr. VÁzquez Domínguez, Carmen: «Las escuelas normales del siglo xix: la formación del profesorado en Cádiz», Tavira, Cádiz, n. ${ }^{\circ} 26$ (2010), pp. 25-53.

${ }_{28}$ Cortes Constiturentes: Proposición de Ley, del Sr. D. Manuel Becerro, sobre primera enseñanza para España y sus islas adyacentes. Acta de la sesión de in de diciembre de i870, Apéndice primero al n. ${ }^{\circ} 320$, I870, pp. I-I8.

${ }_{29}$ Ávila Fernández, A. y Holgado Barroso, J. A.: Formación del Magisterio en España. La legislación normalista como instrumento de poder y control (1834-2007), Madrid, Ministerio de Educación, Política Social y Deporte, 2008, p. 93.

30 Ministerio de Fomento: «Real Orden de 24 de agosto de i878, disponiendo que se comprenda en el cuadro de asignaturas de las Escuelas Normales Centrales de Maestros y Maestras las de Música y Canto», Gaceta de Madrid de io de septiembre de I878, n. ${ }^{\circ} 253$, p. 730.

${ }^{31}$ Agustín M. Lerate, sevillano, profesor de la Real Academia Filarmónica de Sta. Cecilia y maestro de música de la Escuela Normal de Cádiz.

32 Lerate, Agustín M.: Abecedario musical ó Cartilla de Música y Canto: escrita expresamente para Escuelas de Primera enseñanza, Cádiz, Imprenta de Constancia, I884, p. I. 
LA CANCIÓN ESCOLAR EN ESPAÑA ENTRE I9O0 Y I936. CONTRIBUCIÓN AL ESTUDIO HISTÓRICO DE LA EDUCACIÓN MUSICAL CONSUELO DE LA VEGA SESTELO

\subsection{Plan de estudios de 1898}

La realidad social, política e ideológica que vive España en el último tercio del siglo xIx, tal como nos comentan Ávila y Holgado ${ }^{33}$ era la ruptura total y definitiva de la hegemonía ideológica del bloque oligárquico, estando así ante una de las etapas históricas donde existe una mayor identificación e interconexión ideológicas entre cultura y educación. Los males del país necesitan remedios que rectifiquen su trayectoria social; la educación será utilizada como elemento clave y dinamizador para ese desarrollo acometiendo una reforma de las Escuelas Normales y de sus estudios $^{34}$. Estos centros sufrían graves problemas, viviendo hasta esos momentos con la reglamentación de i847. Para esta reforma se tiene en cuenta aspectos de organización: la implantación de la asignatura de Música y Canto en países como Alemania, estudios pedagógicos realizados en nuestro país hasta ese momento y antecedentes legales. Es evidente la influencia de la Institución Libre de Enseñanza, tal como afirma Melcón Beltrán ${ }^{35}$, cuyos miembros tuvieron oportunidad de hacer oír su voz en los círculos gubernamentales con la llegada de los liberales al poder.

Muchas son las voces que consideran que la reforma de 1898 no resuelve las lamentables actuaciones y violaciones de la legislación que se están produciendo. Como ejemplo un comentario en el periódico Nuevo Diario de Badajoz ${ }^{36}$ :

[...] En todos los órdenes de la Administración española estamos cansados de ver anomalías y cosas raras, pero se nos antoja que nada hay tan disparatado como la reforma que recientemente han sufrido las Escuelas Normales, reforma que prueba por medio elocuente que en nuestro país se legisla con absoluto desconocimiento de la realidad, o como quien dice, a ojo de buen cubero.

Ante estas consideraciones podemos decir que la Música y Canto son tratadas como materias propias del plan de estudios, pero que con los mismos problemas y avatares que las demás asignaturas. Este nuevo plan solo tuvo un año de duración.

\subsection{Plan de estudios de 1900}

En este año fue suprimido el Ministerio de Fomento creándose dos nuevos departamentos: el Ministerio de Instrucción Pública y Bellas Artes y el Ministerio de Agricultura, Industria, Comercio y Obras públicas ${ }^{37}$. Ante esta nueva

33 Ávila y Holgado: op. cit., p. iI3.

34 Ministerio de Fomento: «Real Decreto de 23 de septiembre de i898, reformando las Escuelas Normales en la forma que se expresa», Gaceta de Madrid de 25 de septiembre de 1898, n. ${ }^{\circ}$ 268, pp. I25I-I257.

${ }_{35}$ Cfr. Melcón Beltrán, Julia: La formación del profesorado en España (I837-I9I4), Madrid, Ministerio de Educación y Ciencia, 1992, p. 66.

36 Nuevo Diario de Badajoz de 3 de febrero de 190o, p. 2, col. 4. ${ }^{\text {a }}$. Texto recogido en: Cobos Bueno, José M.a: Escuela Normal de Badajoz. Apuntes documentales para su historia, Badajoz, Diputación Provincial, Departamento de Publicaciones, 1996, p. 49.

37 Presidencia del Consejo de Ministros: «Real Decreto de i 8 de abril de 1900 , suprimiendo el Ministerio de Fomento y creando en su lugar los de Instrucción pública y Obras públicas», Gaceta de Madrid de i9 de abril de 1900, n. ${ }^{\circ}$ I09, pp. 316-317. 
LA CANCIÓN ESCOLAR EN ESPAÑA ENTRE I9OO Y 1936. CONTRIBUCIÓN AL ESTUDIO HISTÓRICO DE LA EDUCACIÓN MUSICAL CONSUELO DE LA VEGA SESTELO

distribución administrativa nace un nuevo plan de estudios ${ }^{38}$ del Magisterio, proyecto reformista, en parte similar al de I898, al cual sucederían otros. Introduce nuevas reformas consideradas como trascendentales para poner término a la situación en la que seguían encontrándose las Escuelas Normales, entre otras, se simplifican las asignaturas. Se incluye la Música en los dos cursos superiores, aplicando esta enseñanza en particular al canto coral.

\subsection{Los planes de estudio de I9oI, I903 y I905}

En I90I los problemas económicos continúan. Se reforman los Institutos ${ }^{39}$ para que no solo atiendan la segunda enseñanza, sino que además se ocupen de las enseñanzas técnicas del Magisterio, entre otras. Desaparece la clase de Maestro Normal, quedando la carrera de Magisterio solamente con dos grados: elemental y superior. La Música desaparece de este plan de estudios. Esta reforma se anula, en gran parte, días después ${ }^{40}$ reflejando lo que eran estos años, con continuas aprobaciones y anulaciones de disposiciones. En $1902^{4 \mathrm{I}}$ se restaura la asignatura de Música en las Normales Superiores para, en ese mismo año, incluirla como obligatoria en los planes de estudio.

El plan de estudios de $1903^{42}$ nace con la necesidad de una reforma fundamentada en el número extraordinario de escuelas públicas vacantes y no solicitadas, debido, en parte, a la escasa retribución de que disfrutaban los maestros. La asignatura de Música solo se encuentra en el título superior de maestro.

En 1905 se aprueba un Real Decreto ${ }^{43}$ que organiza de nuevo las Escuelas Normales. Este plan de estudios no llegaría a implantarse por falta de recursos económicos. En él se daba gran importancia a la enseñanza del Dibujo y de la Música y Canto siguiendo lo expuesto en los últimos Congresos Internacionales.

38 Ministerio de Instrucción Pública y Bellas Artes: «Real Decreto de 6 de julio de 1900, modificando la actual organización de las Escuelas Normales y de la Inspección de primera enseñanza», Gaceta de Madrid de 8 de julio de I900, n. ${ }^{\circ}$ I89, pp. II3-II5.

39 Ministerio de Instrucción Pública y Bellas Artes: «Real Decreto de i7 de agosto de I9oI, reformando los estudios de segunda enseñanza y las enseñanzas técnicas del Magisterio, Industria, Comercio, Bellas Artes y Artes Industriales», Gaceta de Madrid I9 de agosto de I90I, n. ${ }^{\circ}$ 23I, pp. 790-795.

${ }_{40}$ Ministerio de Instrucción Pública y Bellas Artes: «Real Orden de 26 de agosto de i9oi, disponiendo la forma en que han de hacerse los estudios de las carreras de Maestros y Maestras ínterin se ponen en vigor los preceptos del Real Decreto de 17 del actual», Gaceta de Madrid de 28 de agosto de I9OI, n. ${ }^{\circ} 240$, p. IO52.

${ }_{41}$ Subsecretaría del Ministerio de Instrucción Pública y Bellas Artes: «Disposición de iz de enero de 1902, para que den comienzo desde luego las clases de Música en las Escuelas Normales Superiores", Gaceta de Madrid de I5 de enero de I902, n. ${ }^{\circ}$ I5, p. 212.

42 Ministerio de Instrucción Pública y Bellas Artes: «RealDecreto de 24 de septiembre de 1903 , determinando la forma en que han de efectuarse desde el I. ${ }^{\circ}$ del próximo Octubre los estudios del Magisterio elemental», Gaceta de Madrid de 26 de septiembre de 1903, n. ${ }^{\circ} 269$, pp. 2560-256I.

43 Ministerio de Instrucción Pública y Bellas Artes: «Real Decreto de 30 de marzo de 1905, reorganizando las Escuelas Normales», Gaceta de Madrid de 3I de marzo de 1905, n. ${ }^{\circ} 90$, pp. I215-1217. 
LA CANCIÓN ESCOLAR EN ESPAÑA ENTRE I9OO Y I936. CONTRIBUCIÓN AL ESTUDIO HISTÓRICO DE LA EDUCACIÓN MUSICAL CONSUELO DE LA VEGA SESTELO

\subsection{Los avatares y movimientos de la legislación. El plan de estudios de I9I4}

Anterior al plan de I9I4 se suceden intentos normativos y administrativos para solucionar las grandes deficiencias que existen en la enseñanza, sobre todo primaria y normal y en el carácter urgente de atender y elevar el nivel de la cultura del país. Como ejemplo el proyecto de Ley orgánica de Instrucción Pública ${ }^{44}$ autorizado para someterlo a la deliberación de las Cortes, en 1905. En él, los estudios de las Escuelas Normales se organizarán en cuatro cursos. En los tres primeros se incluye la de Música y Canto.

En la Gaceta de Madrid del 8 de diciembre de i9ıo se publica una Real Orden ${ }^{45}$ disponiendo que la enseñanza de la Música en las Escuelas Normales Superiores, tanto de Maestras como de Maestros, se ajuste al plan propuesto por el Conservatorio Nacional de Música y Declamación. Sus contenidos:

Primer curso. Comprende: «El conocimiento de las notas, figuras, silencios, pentágrama, líneas adicionales, claves, compases de compasillo, 2 por 4 y 3 por 4 , partes fuertes y débiles, escala diatónica mayor y menor, tonos y semitonos de que consta, puntillo, ligadura, calderón, síncopa, sostenido, bemol y becuadro, intervalos, conjunto y disjunto, mayores y menores, movimientos del compás ó aires. En las lecciones prácticas de este año se hace uso del tono de Do mayor y de La menor en clave de sol. Compases de compasillo, 2 por 4 y 3 por 4, síncopa y puntillo hasta la corchea y semicorchea y su silencio. Se puede empezar á aprender algún canto escolar fácil, de una sola voz».

Segundo curso. Comprende: «Conocimiento de los tonos y modos mayores y menores hasta tres sostenidos y tres bemoles, alteraciones propias y accidentales, compás binario, 3 por 8,6 por 8,9 por 8 y I2 por 8 . Apoyaturas y mordentes, doble puntillo, seisillo y sus silencios, fusas, clasificación en los compases de combinación doble y triple. Conocimiento de la clave de $\mathrm{Fa}$ en cuarta línea. En las lecciones prácticas se hace uso de los tonos mayores y menores mencionados y de todas las combinaciones de medida hasta la fusa. Habiendo practicado los cantos escolares á una voz, pueden adiestrarse con los de dos voces».

Si resumimos los contenidos que se expusieron, nos encontramos con aspectos teóricos, rítmicos, de lectura y entonación; el trabajo teórico y práctico a través de ejercicios y de canciones escolares a una y dos voces. No se incluyen contenidos instrumentales y tampoco referidos a la Didáctica y Metodología de trabajo. Esta programación de la asignatura es de gran importancia estando en vigor a lo largo de este periodo, hasta 1936.

44 Ministerio de Instrucción Pública y Bellas Artes: «Real Decreto de i4 de junio de 1905, autorizando al Ministro de Instrucción Pública para someter á deliberación de las Cortes un proyecto de ley orgánica de instrucción primaria», Gaceta de Madrid de 16 de junio de 1905, n. ${ }^{\circ}$ 167, pp. 1089-1092.

${ }_{45}$ Ministerio de Instrucción Pública y Bellas Artes: «Real Orden de is de noviembre de I9ıo, disponiendo que la enseñanza de la Música en las Escuelas Normales Superiores de Maestros y Maestras se ajuste al plan propuesto por el Conservatorio Nacional de Música y Declamación», Gaceta de Madrid de 8 de diciembre de I910, n. ${ }^{\circ} 342$, p. 570. 
LA CANCIÓN ESCOLAR EN ESPAÑA ENTRE I9OO Y I936. CONTRIBUCIÓN AL ESTUDIO HISTÓRICO DE LA EDUCACIÓN MUSICAL CONSUELO DE LA VEGA SESTELO

En i9r3 la Dirección General de Primera enseñanza acuerda ${ }^{46}$ realizar cursos de perfeccionamiento y ampliación de estudios para Maestros y Maestras de primera enseñanza oficial. Entre otros contempla clases prácticas acerca de las materias del programa escolar e iniciación a los métodos de dibujo, canto y juegos escolares.

En I9I4 se realiza una reforma de las Escuelas Normales ${ }^{47}$ denominada plan Bergamín por el ministro que lo suscribió, muy importante en la época y con una larga vigencia, hasta el plan de estudios de 193I. Se adquiere cierta estabilidad en la formación del profesorado, después de los diversos planes de estudio que se venían sucediendo. Los estudios se distribuirán a lo largo de cuatro cursos académicos. Entre las materias del primer y segundo curso se encuentra la Música. Estos estudios tendrán un carácter eminentemente educativo, atendiendo no solo a impartir los contenidos que se establecen, sino también a despertar la iniciativa de los alumnos, desarrollando en ellos la observación y la reflexión, y realizando aplicaciones prácticas de la doctrina enseñada. Las clases serán de hora y media de duración, excepto las de Labores que durarán dos horas. En el caso de Música estas clases serán bisemanales.

\subsection{Los planes de estudios de 1931}

Esta reforma, llevada a cabo por la República, intenta hacer del maestro o maestra el alma de la Escuela, fortaleciendo, independizando, sosteniendo y formando su propia alma. Esta reforma de $1931^{48}$ pretende ser a la vez social, cultural y económica. Establece dos planes de estudios: uno permanente de preparación del Magisterio primario y otro transitorio de cultura general. En la preparación permanente las disciplinas abarcarán: conocimientos filosóficos, pedagógicos y sociales; metodologías especiales; y materias artísticas y plásticas. Se distribuyen en tres cursos y la Música se estudiará en primero y segundo. En el plan transitorio los estudios se distribuyen en tres cursos. En el tercero se incluye la asignatura de Música y Canto.

46 Ministerio de Instrucción Pública y Bellas Artes: «Real Orden de 28 de marzo de i9i3, disponiendo se organicen, donde la Dirección General de Primera enseñanza acuerde, cursos de perfeccionamiento y ampliación de estudios para Maestros y Maestras de primera enseñanza oficial», Gaceta de Madrid de 3I de marzo de I9I3, n. ${ }^{\circ}$ 90, p. 807.

47 Ministerio de Instrucción Pública y Bellas Artes: «Real Decreto de 30 de agosto de i9i4, relativo a la reorganización de las Escuelas Normales de Primera enseñanza», Gaceta de Madrid de 2 de septiembre de i914, n. ${ }^{\circ} 245$, pp. $562-567$.

${ }_{48}$ Ministerio de Instrucción Pública y Bellas Artes: «Decreto de 29 de Septiembre de r93I, dictando normas relativas a la preparación del Magisterio primario», Gaceta de Madrid de 30 de septiembre de I931, n. ${ }^{\circ} 273$, pp. 209I-2094. 
LA CANCIÓN ESCOLAR EN ESPAÑA ENTRE I9O0 Y I936. CONTRIBUCIÓN AL ESTUDIO HISTÓRICO DE LA EDUCACIÓN MUSICAL CONSUELO DE LA VEGA SESTELO

\section{Análisis de las canciones escolares y métodos de música para Escuelas Normales en España entre 1900 y 1936}

\section{I. Planteamiento metodológico de la investigación}

Corresponden los resultados ofrecidos a una investigación ${ }^{49}$ realizada por medio del análisis de 782 canciones infantiles y escolares pertenecientes al periodo de 1900 y 1936 en España o utilizadas en él, de 42 autores distintos. Se estudian 36 manuales de música para Escuelas Normales correspondientes a 24 autores.

Desde el punto de vista metodológico se utilizan aspectos de la investigación histórica, aplicada siempre a la historia de la educación y aspectos de la investigación musical en sus facetas histórica, didáctica y analítica. Investigación histórica por la época de estudio, entre 1900 y 1936, no considerada contemporánea a nosotros, con ideologías, costumbres, pensamientos, manifestaciones y hechos que forman parte de nuestro acervo cultural, social, político y educativo. Musical por los tipos de materiales analizados. El estudio de estos materiales, la comprensión de sus autores, de sus enseñantes, de sus principios musicales nos darán herramientas de entendimiento de procesos, pensamientos, hechos y circunstancias en torno a una parcela de la educación musical. Didáctica por la «zona» de acción, la educación primaria y educación en Escuelas Normales, limitándolo a la enseñanza de la Música y a la utilización de las canciones infantiles y escolares.

El trabajo ha sido delimitado, en un primer nivel de concreción, a la educación primaria y educación en Escuelas Normales en España entre I900 y 1936. Como segundo nivel de concreción al estudio y análisis de las canciones infantiles y escolares y de los métodos de música empleados en Escuelas Normales. Estos dos niveles dan lugar a la utilización de distintos enfoques metodológicos y al empleo de un método de trabajo apropiado a las ciencias sociales, a la historia de la educación, a los textos referidos y al área artística, Música. Estos enfoques se centran en la utilización de los métodos descriptivo, analítico, de síntesis, comparativo y hermenéutico llegando al intento de conocimiento que pretende explicar las relaciones existentes entre los aspectos que han motivado la realización de los textos y composiciones publicadas, las inquietudes de los autores, los aspectos sociales de la época y relaciones con el entorno.

Para la toma de datos se han desarrollado dos vertientes: por un lado, se han definido las unidades de análisis sobre las que se centra este proceso; en segundo lugar, se han elaborado las fichas correspondientes a cada unidad de análisis establecida.

Las unidades de análisis son: a) la canción escolar en torno a la cual se realiza un proceso de análisis y toma de datos. Se atienden aspectos melódicos, rítmicos, armónicos, formales, de acompañamiento instrumental, agógicos y dinámicos, sobre el texto y didácticos; b) método de música destinado a Escuelas Normales, estudio de cada obra como un todo: estructura y organización del manual, contenidos y secuenciación, y aspectos didácticos y metodológicos.

49 De La Vega Sestelo, M. C.: La canción escolar en España entre rgoo y 1936, tesis doctoral no publicada, director Dr. José M. ${ }^{a}$ Hernández Díaz, Salamanca, Universidad de Salamanca, Departamento de Teoría e Historia de la Educación, zorz. 
LA CANCIÓN ESCOLAR EN ESPAÑA ENTRE I9OO Y 1936. CONTRIBUCIÓN AL ESTUDIO HISTÓRICO DE LA EDUCACIÓN MUSICAL CONSUELO DE LA VEGA SESTELO

Se elaboran fichas de análisis diferentes, una para cada unidad de análisis. Se determinan las categorías de análisis después de la toma de datos correspondiente. Para la elaboración de las categorías se han tenido en cuenta aspectos musicales y extramusicales que se relacionan entre sí. En cada agrupamiento, las dificultades didácticas y de enseñanza que conllevan nos ayudan a establecer «intervalos» de contenidos o criterios como son: tipos y presentación de los contenidos, dificultades de aprendizaje y criterios compositivos.

Para el tratamiento de los datos obtenidos y obtención de conclusiones, se ha considerado: en el análisis de canciones infantiles y escolares la utilización de porcentajes, dado que el volumen de canciones que se manejan es suficiente para poder extrapolar estos datos a una generalidad mayor. Debido a la naturaleza diversa en estructura, contenidos y formas de tratarlos, de los métodos de música para Escuelas Normales, se opta por una interpretación y reflexión sobre los datos obtenidos y así establecer puntos para un análisis comparativo posterior. Se han introducido factores comparativos que nos ponen en relación las canciones escolares con los métodos de música empleados en las Escuelas Normales, y todos ellos con el plan de estudios de igro para la enseñanza de la Música en estos establecimientos.

\subsection{Resultados obtenidos}

Se ha realizado un trabajo de recopilación tanto de canciones infantiles escritas con fines escolares como de obras destinadas a la enseñanza de la música en Escuelas Normales. En total se han estudiado 782 canciones, pero se han podido registrar, aproximadamente, Ioo canciones más y 36 métodos para Escuelas Normales (se ha encontrado la referencia de i4 obras más que no han sido localizadas).

Del análisis y posterior tratamiento de los datos de las canciones infantiles y escolares se establecen unas características compositivas como las más comunes utilizadas por los compositores para este tipo de obras. Estas características corresponden tanto a aspectos de sencillez, de tipos de voces a los que van dirigidas, a aspectos psicológicos por la edad de sus destinatarios, como a aspectos pedagógicos y didácticos que tienen que ver con los procesos de enseñanza y aprendizaje. Se resumen en la siguiente tabla:

TABLA I: Características compositivas y musicales más comunes en las canciones

Características compositivas y musicales más comunes en las canciones

\begin{tabular}{|c|l|}
\hline Escritura & Clave de sol en la escritura \\
\hline \multirow{4}{*}{ Ámbito y extensión } & Extensión aproximada de las canciones entre $\mathrm{I}_{3}$ y 48 compases \\
\cline { 2 - 2 } & $\begin{array}{l}\text { Utilización de la misma melodía para distintas estrofas del texto que facilita } \\
\text { su aprendizaje }\end{array}$ \\
\cline { 2 - 2 } & Ámbito aproximado entre $\mathrm{La}_{3} \mathrm{y} \mathrm{Mi}_{5}$ \\
\hline
\end{tabular}


LA CANCIÓN ESCOLAR EN ESPAÑA ENTRE I9OO Y 1936. CONTRIBUCIÓN AL ESTUDIO HISTÓRICO DE LA EDUCACIÓN MUSICAL CONSUELO DE LA VEGA SESTELO

\begin{tabular}{|c|c|}
\hline \multicolumn{2}{|c|}{ Características compositivas y musicales más comunes en las canciones } \\
\hline \multirow{6}{*}{ Aspectos rítmicos } & $\begin{array}{l}\text { Preferencia de la utilización de compases de subdivisión binaria frente a los } \\
\text { de subdivisión ternaria }\end{array}$ \\
\hline & Compases más usados: los simples de denominador 4 \\
\hline & $\begin{array}{l}\text { Figuras básicas: blanca, negra, corchea y sus silencios. La semicorchea y } \\
\text { silencio se encuentra en grupos rítmicos específicos que imprimen a las } \\
\text { composiciones características rítmicas concretas }\end{array}$ \\
\hline & $\begin{array}{l}\text { Se emplean elementos rítmicos como la ligadura de prolongación, el } \\
\text { puntillo, la síncopa y el contratiempo }\end{array}$ \\
\hline & Grupo de valoración especial más común: tresillo \\
\hline & Inclusión de notas de adorno, solo de forma testimonial \\
\hline \multirow{5}{*}{ Aspectos melódicos } & $\begin{array}{l}\text { Preferencia de tonalidades mayores frente a las menores y hasta } 4 \text { alteraciones } \\
\text { en la armadura }\end{array}$ \\
\hline & $\begin{array}{l}\text { Poco empleo de alteraciones accidentales, su aparición se realiza más fre- } \\
\text { cuentemente como floreos, cromatismos y alteración del } 6 .^{\circ} \text { y } 7 .^{\circ} \text { grados del } \\
\text { modo menor }\end{array}$ \\
\hline & $\begin{array}{l}\text { Uso, casi en exclusividad, de intervalos mayores, menores y justos, de forma } \\
\text { simple y no compuesta }\end{array}$ \\
\hline & $\begin{array}{l}\text { Cambios de tonalidad y modulaciones más utilizadas: a tonos vecinos, } \\
\text { homónimo y tono de la dominante }\end{array}$ \\
\hline & Cadencia perfecta como cadencia final principal \\
\hline \multirow{7}{*}{ Aspectos formales } & $\begin{array}{l}\text { Formas musicales músico elementales a excepción del canon, no encontrado } \\
\text { en ninguna canción }\end{array}$ \\
\hline & Formas de comienzo de la melodía más empleadas: tético y anacrúsico \\
\hline & Final de la melodía más empleado: masculino \\
\hline & Utilización masiva de frases musicales regulares frente a las irregulares \\
\hline & Extensión de las frases musicales más frecuente: 8 compases \\
\hline & Estructura binaria como estructura interna de las frases más utilizada \\
\hline & Signos de repetición \\
\hline \multirow[t]{2}{*}{ Aspectos expresivos } & $\begin{array}{l}\text { Términos de tempo, agógicos, dinámicos y en menor medida de carácter, } \\
\text { incluyendo terminología básica y sencilla desde el punto de vista didáctico } \\
\text { que refleja matices bien diferenciados }\end{array}$ \\
\hline & $\begin{array}{l}\text { Signos de articulación y acentuación más empleados: ligadura de expresión, } \\
\text { coma de respiración y acentos }\end{array}$ \\
\hline Instrumentación & $\begin{array}{l}\text { Mayoritariamente compuestas con acompañamiento instrumental para piano } \\
\text { o armonium }\end{array}$ \\
\hline
\end{tabular}

Fuente: Elaboración propia. 
LA CANCIÓN ESCOLAR EN ESPAÑA ENTRE I9O0 Y I936.

CONSUELO DE LA VEGA SESTELO

El análisis de los textos de las canciones nos ofrece datos sobre la educación de los niños y lo que la música puede contribuir en ello. Con el texto creado o tomado de otros autores se buscan los siguientes objetivos: educación moral y religiosa, educación patriótica, formación en conocimientos, educación en costumbres y tradiciones, la vida cotidiana y el entorno del niño, así como el amor por la naturaleza.

Los métodos de música y manuales para Escuelas Normales se organizan en teóricos y prácticos. Los métodos teóricos incluyen, en su mayoría, los siguientes contenidos básicos (no siempre con la misma profundidad) expuestos en la siguiente tabla:

Tabla II: Contenidos teóricos de los métodos para las Escuelas Normales

\begin{tabular}{|c|c|}
\hline \multicolumn{2}{|r|}{ Contenidos teóricos de los métodos para las Escuelas Normales } \\
\hline $\begin{array}{l}\text { Contenidos } \\
\text { iniciales }\end{array}$ & $\begin{array}{l}\text { Aspectos generales: música, solfeo, pentagrama, notas, clave de Sol y Fa en } \\
\text { 4. }^{\text {a }} \text { líneas adicionales y líneas divisorias }\end{array}$ \\
\hline \multirow{7}{*}{$\begin{array}{l}\text { Contenidos } \\
\text { armónicos, } \\
\text { melódicos } \\
\text { y formales }\end{array}$} & Tonalidades, escalas relativas, escalas menores (armónica y melódica) \\
\hline & Escala cromática \\
\hline & Alteraciones propias y accidentales \\
\hline & Tono y semitono (diatónico y cromático) \\
\hline & Intervalos, clasificación e inversión \\
\hline & Armonía: concepto de acorde (tratados de forma minoritaria) \\
\hline & Melodía y frase musical \\
\hline \multirow{6}{*}{$\begin{array}{l}\text { Contenidos } \\
\text { rítmicos }\end{array}$} & $\begin{array}{l}\text { Compás: forma de marcarlos en el espacio, clasificación, subdivisión, } \\
\text { comienzo incompleto (anacrúsico) }\end{array}$ \\
\hline & Figuras, silencios y su relación \\
\hline & Síncopas y notas a contratiempo \\
\hline & Puntillos y ligaduras de prolongación \\
\hline & Grupos de valoración especial [denominación actual] \\
\hline & Notas de adorno \\
\hline \multirow{3}{*}{$\begin{array}{l}\text { Contenidos } \\
\text { sobre expresión }\end{array}$} & $\begin{array}{l}\text { Términos de tempo, carácter, agógicos, dinámicos y signos de articulación y } \\
\text { acentuación. El calderón }\end{array}$ \\
\hline & Signos de repetición \\
\hline & El metrónomo \\
\hline $\begin{array}{l}\text { Contenidos } \\
\text { vocales }\end{array}$ & $\begin{array}{l}\text { Aspectos vocales: respiración y vocalización, clasificación de las voces, la } \\
\text { canción escolar (tratados de forma minoritaria) }\end{array}$ \\
\hline
\end{tabular}

Fuente: Elaboración propia.

Los métodos prácticos emplean las siguientes dificultades para la realización de los ejercicios resumidos en la siguiente tabla explicativa: 
LA CANCIÓN ESCOLAR EN ESPAÑA ENTRE I9OO Y 1936. CONTRIBUCIÓN AL ESTUDIO HISTÓRICO DE LA EDUCACIÓN MUSICAL CONSUELO DE LA VEGA SESTELO

Tabla III: Dificultades prácticas en los métodos para las Escuelas Normales

\begin{tabular}{|c|c|}
\hline \multicolumn{2}{|r|}{ Dificultades prácticas en los métodos para las Escuelas Normales } \\
\hline \multirow{4}{*}{$\begin{array}{l}\text { Aspectos } \\
\text { melódicos }\end{array}$} & $\begin{array}{l}\text { Escalas mayores y, en menor grado, menores de hasta } 3 \text { alteraciones en la } \\
\text { armadura }\end{array}$ \\
\hline & Tipos de escalas menores utilizadas: armónica y melódica \\
\hline & $\begin{array}{l}\text { Uso de alteraciones accidentales como: floreos, cromatismos y } 6 .^{\circ} \text { y } 7 .^{\circ} \\
\text { grados del modo menor }\end{array}$ \\
\hline & Empleo de intervalos mayores, menores y justos en su forma simple \\
\hline \multirow{4}{*}{$\begin{array}{l}\text { Aspectos } \\
\text { rítmicos }\end{array}$} & $\begin{array}{l}\text { Compases más relevantes en los ejercicios: compases simples de denominador } \\
4 \text { y compuestos de denominador } 8\end{array}$ \\
\hline & $\begin{array}{l}\text { En lectura rítmica: figuras hasta la semicorchea y su silencio, en mucha } \\
\text { menor medida la fusa (empleada en ejercicios con subdivisión) }\end{array}$ \\
\hline & $\begin{array}{l}\text { Grupos rítmicos que incluyen: síncopas, notas a contratiempo, puntillo y } \\
\text { doble puntillo, ligaduras de prolongación } \\
\text { Grupos de valoración especial: tresillo, seisillo, dosillo y cuatrillo }\end{array}$ \\
\hline & $\begin{array}{l}\text { Notas de adorno básicas en la lectura: apoyaturas y mordentes de I, } 2 \text { y } 3 \\
\text { notas }\end{array}$ \\
\hline \multirow{4}{*}{$\begin{array}{l}\text { Aspectos } \\
\text { expresivos }\end{array}$} & $\begin{array}{l}\text { Utilización de términos de tempo, carácter (de forma escasa), términos } \\
\text { agógicos y dinámicos }\end{array}$ \\
\hline & $\begin{array}{l}\text { La ligadura de expresión como articulación y fraseo. El calderón. Signos de } \\
\text { repetición }\end{array}$ \\
\hline & Uso de indicaciones metronómicas (forma testimonial) \\
\hline & Ejercicios de vocalización, respiración y articulación (forma testimonial) \\
\hline
\end{tabular}

Fuente: Elaboración propia.

\section{Conclusiones}

Podemos concluir para este periodo de 1900 a 1936 en España que:

La normativa solo incluye la realización de cantos sencillos en la enseñanza primaria, no preocupándose en todo el periodo de modificaciones o actualización. Al igual que las demás materias, la música sufre una situación precaria y de abandono, siendo considerada o no considerada, dependiendo de las circunstancias, como materia a incluir en los múltiples intentos de reforma.

La Música se incluye en gran parte de los planes de estudio de las Escuelas Normales aunque con distinto tratamiento. Era de las asignaturas de posible supresión si los motivos económicos lo determinaban, siempre impartida por un profesor especial, peor pagado que la mayoría.

Se puede afirmar que la formación de los profesores especiales que ejercían su docencia en las Escuelas Normales, tanto a nivel musical como didáctico, era muy 
LA CANCIÓN ESCOLAR EN ESPAÑA ENTRE I9OO Y I936. CONTRIBUCIÓN AL ESTUDIO HISTÓRICO DE LA EDUCACIÓN MUSICAL CONSUELO DE LA VEGA SESTELO

buena. Dato que se puede verificar en las pruebas y oposiciones convocadas para tal fin. Si existen problemas en la formación musical de los maestros no creemos que fuera debido a una deficiente preparación de los profesores especiales sino al poco tiempo y cursos que se dedicaban a esta materia.

El volumen de publicaciones encontradas nos deja constancia de la importancia que en la época se daba a la canción en la educación primaria en España desde los sectores más implicados en ello. Encontramos nombres de músicos muy considerados como: Eduardo Martínez Torner, Rafael Benedito, Ricardo Boronat, Amancio Amorós, Emilio Ramírez, Josep Cumellas, Ruperto Chapí, José M. Franco, Manuel Borguñó, Rodolfo Halffter, José Salvador Martí, etc., que demuestra la importancia que, en la formación musical de la población, se le daba a la enseñanza de canciones en la escuela.

Todo esto no tuvo correspondencia en el desarrollo legislativo de los planes para las escuelas primarias. Aunque reconocido en el plan de estudios de I90I, la práctica de canciones no fue generalizada siendo reflejo de las deficiencias que estas escuelas sufrían en la época y su necesidad de reforma. Se planteaba la formación musical como actividad lúdica con cantos sencillos que ayudaban al niño a cambios de materias consideradas más importantes en la concepción escolar.

Didácticamente los compositores de las canciones se preocupaban por aspectos que facilitasen su enseñanza:

- En la escritura rítmica, el pulso rítmico puede ser apreciado con suma facilidad.

- Se emplea, preferentemente, la relación de una nota por cada sílaba del texto.

- Los intervalos empleados suelen ser inferiores a la 6. ${ }^{\mathrm{a}}$, utilizando el grado conjunto con mucha más frecuencia que el disjunto, por su menor dificultad de aprendizaje.

- Tempos moderados o rápidos, evitando los muy lentos, lentos o muy rápidos que añadirían un grado de dificultad de aprendizaje en los niños; su necesidad de juego y movimiento contempla una tendencia a pulsos de velocidades moderadas o rápidas.

- La armonía en las composiciones se basa en afianzar el sentido tonal.

- Los textos son escogidos o creados con minuciosidad en temas, palabras empleadas y dificultades lingüísticas, pensando en todo momento que van destinados a la niñez y lo que esto significaba en el primer tercio del siglo $\mathrm{xx}$. Se elige la escritura en verso antes que en prosa por su analogía rítmica, a excepción de los cantos religiosos.

Con el análisis de las canciones escolares se puede constatar que en este periodo:

- Existe un volumen importante de composiciones destinadas al mundo escolar.

- Parte de los compositores de la época invierten tiempo en estas composiciones.

- La mayoría de los autores son a la vez docentes, interesados por novedades y avances pedagógicos. 
- Todo el material centra en la canción infantil el elemento más importante de la formación musical de los niños, siendo, a la vez, el núcleo metodológico y el objetivo del aprendizaje.

- Existen características compositivas comunes en las canciones infantiles y escolares, que aun no siendo lo suficientemente consideradas como para establecer un género musical sí sirven para diferenciar este tipo de composiciones con unos destinatarios y fines muy concretos.

- Muchos músicos de la época no son ajenos a las corrientes pedagógicas que «apuestan» por una educación estética de la infancia donde la música tendría un gran peso en esa educación.

- Aunque no hemos encontrado demasiadas afirmaciones sobre la adecuación de estas composiciones a un plan de estudios en concreto, sí hemos observado que en gran parte de las canciones compuestas con posterioridad a I9Io se intenta respetar los contenidos que para la materia de Música en las Escuelas Normales establece el programa de esta asignatura aprobado en ese mismo año.

Los textos de música destinados a la enseñanza en Escuelas Normales o con sospecha de poder haber sido utilizados para tal fin son de dos tipos: teóricos y prácticos. El objetivo perseguido por unos u otros es distinto, mientras con los teóricos se pretendía la formación en este tipo de contenidos, que suponía un «estilo» y metodología de aprendizaje más memorístico, con los prácticos el objetivo era enseñar la lectura, escritura e interpretación de obras o composiciones que utilizan el lenguaje musical como «idioma» de la música. De su análisis podemos obtener las siguientes conclusiones:

- La procedencia de los autores, al igual que ocurre en los compositores de las canciones, se circunscribe, en una mayoría, al noreste de España y a Madrid.

- Son 24 los autores estudiados. De ellos tenemos constancia de que 9 fueron profesores especiales de música en Escuelas Normales. Todos los demás autores de los que poseemos datos estaban relacionados con la docencia musical en escuelas de primaria u otros centros educativos.

- A pesar de que los textos son teóricos y prácticos, la mayoría de estos autores consideraban que la enseñanza de la Música debía basarse en la práctica, tanto de lectura musical, interpretación vocal y dentro de lo posible instrumental (piano), poniendo la teoría al servicio de la práctica.

- Toda la enseñanza de música en las Escuelas Normales iba enfocada al aprendizaje, interpretación y forma de enseñanza de canciones infantiles y escolares con aplicación en la enseñanza primaria. Los ejercicios prácticos realizados estaban pensados en su mayoría para ser leídos y entonados a la vez.

Desde el punto de vista didáctico de estos manuales podemos comentar:

- Las obras están escritas pedagógicamente. Con un orden específico en la aparición de los contenidos, en las dificultades planteadas, con una estructura de lecciones, temas o secciones. No son obras de simple divulgación; 
LA CANCIÓN ESCOLAR EN ESPAÑA ENTRE I9OO Y 1936.

CONTRIBUCIÓN AL ESTUDIO HISTÓRICO DE LA EDUCACIÓN MUSICAL CONSUELO DE LA VEGA SESTELO

su estructura, ordenación, inclusión de ejercicios, ejemplos, etc., obedece a criterios didácticos.

- Los contenidos que se incluyen, en términos generales, son suficientes para abordar las dificultades de lectura, entonación e interpretación de las canciones infantiles y escolares publicadas. Si existen problemas en la preparación del maestro es, sobre todo, debido al poco tiempo de trabajo dedicado a la enseñanza musical en los distintos planes de estudio del magisterio.

- El plan de estudios para la enseñanza de la Música en Escuelas Normales de igio establece los mínimos contenidos que deben recibir los futuros maestros, aunque descuida la formación vocal y pedagógica en el mismo. Los contenidos son coherentes y realistas para poder conseguir una buena formación musical del maestro, pero con poco tiempo y clases para llevarlo a efecto.

- Parte de los autores incluyen indicaciones metodológicas destinadas a la forma de utilizar dicho texto en el aula, desarrollando contenidos metodológicos que sirvan al futuro maestro para poder trabajar las canciones escolares en la escuela primaria.

Como conclusiones finales:

- La educación musical en la enseñanza primaria en España, entre 1900 y 1936, es deficiente. No existe una educación musical como tal; se intenta acercar a la población a la música a través de cantos sencillos en la escuela. En la mayoría de las escuelas no se aplicaba el plan de estudios vigente de I90i que establecía el Canto como materia. Detrás de esta precariedad se pueden encontrar razones económicas, de reestructuración de la enseñanza, de preparación de los maestros, de consideración social de estos docentes, de concepción de lo que la educación significa para la persona y de la consideración, o no, de todos los ciudadanos como iguales.

- Existieron intentos de superación de la situación deficitaria en la que se encontraba la educación musical y, en concreto, general en la enseñanza primaria en España: en el terreno político se suceden proyectos de ley e intentos de reforma; en el ámbito social la Institución Libre de Enseñanza pone en marcha multitud de innovaciones educativas que van demostrando el beneficio que podían aportar a la educación de un país; en ellas se incluye la música, con la práctica del canto coral, audiciones de los grandes autores, conocimiento de los cantos populares, etc. La Guerra Civil española truncará este desarrollo educativo que tantos frutos pudo haber aportado en años sucesivos. En el ámbito profesional el Real Conservatorio de Música y Declamación de Madrid realiza la programación de la asignatura de Música, en i9ıo, para ser aplicada en las Escuelas Normales.

- La formación musical recibida por los maestros era insuficiente. La razón fundamental es el poco tiempo de estudio dedicado a esta materia.

- Conocer lo que ocurrió en épocas anteriores nos ayuda a entender lo que está sucediendo en la actualidad y la búsqueda de nuevas vías de mejora de los problemas estructurales que van surgiendo en la educación. Haciendo 
un pequeño paralelismo entre la época actual y la época que hemos estudiado en España (1900-1936) podemos comentar: la enseñanza musical en la educación primaria actual tiene mayor presencia que en la época expuesta, además es considerada como una materia más y no como una actividad entre las otras materias. Aun así, la enseñanza de la música se encuentra con deficiencias y con una peor consideración. Una de las posibles causas coincide con nuestra historia: la formación musical de los maestros es insuficiente. Los planes de estudios actuales del magisterio no están pensados para la formación técnica y musical centrándose más en los aspectos didácticos y pedagógicos sin exigir ninguna preparación musical para iniciar estos estudios. Existe una necesidad de revisión de los planes de estudio actuales para los maestros en la Mención de Música. 